\title{
Comparison between the lambda response of eye-fixation-related potentials and the P100 component of pattern-reversal visual evoked potentials
}

\author{
KOJI KAZAI and AKIHIRO YAGI \\ Kwansei Gakuin University, Nishinomiya, Hyogo, Japan
}

\begin{abstract}
The purpose of this study was to compare the lambda response of eye-fixation-related potentials (EFRPs) with the P100 component of pattern-reversal visual-evoked potentials. EFRPs were obtained by averaging EEGs time-locked to the offset of the saccade. The dipole of the lambda response and that of the P100 component were estimated by the dipole-tracing method (Musha \& Homma, 1990). The locations of their dipoles at the occipital sites were very close to each other when the difference waveform, which was calculated by subtracting the EFRP to the patternless stimulus from the EFRP to the patterned stimulus, was used for the lambda response. This finding implies that the lambda response and P100 have a common neural generator in the visual cortex. However, the peak latency of the lambda response was shorter than that of P100. The saccades in the EFRP trial were considered to be the cause of the difference.
\end{abstract}

When occipitalEEGs time-locked to the offset of saccadic eye movements (saccades) are averaged, eye-fixationrelated potentials (EFRPs) are obtained (Yagi, 1995). EFRPs are nearly synonymous with the lambda complex (Lesèvre \& Rémond, 1973; Marton \& Szirtes, 1982; Szirtes, Marton, \& Breuer, 1982; Yagi, 1979) or the lambda wave (Billings, 1989; Kurtzberg \& Vaughan, 1973; Morton, Merton, \& Cobb, 1974; Thickbroom, Knezevic, Carroll, \& Mastaglia, 1991). The most prominent component of the EFRP is called the lambda response, which is a sharp positive component with a latency of about $80 \mathrm{msec}$ from the offset of saccades. Some participants show a large deflection identical to the lambda response without the averaging of EEGs (Evans, 1953; Green, 1957; Perez-Borja, Chatrian, Tyce, \& Rivers, 1962; Roth \& Green, 1953; Scott, Groethuysen, \& Bickford, 1967). The lambda response had been considered to occur at the onset of saccades (Kurtzberg \& Vaughan, 1973). Yagi (1979), however, insisted that the lambda response is associated with the offset of saccades. Yagi (1979) compared two kinds of lambda responses: one time-locked to the onset of sac-

This study was supported partly by Grant-in-Aid for Scientific Research 11551003 to A. Y. from the Ministry of Education, Science, Sports, and Culture in Japan and partly by the MITI (Ministry of International Trade and Industry) and NEDO (the New Energy and Industrial Technology Development Organization) Project on Behavior-Based Human Environment Creation Technology. We are grateful to John Jonides and the three anonymous reviewers for critical comments on an earlier draft of the manuscript, to Tetsuya Jibu for making the stimulus for our preliminary experiment, and to Hiroko Fukuda for language assistance. Correspondence concerning this article should be addressed to K. Kazai, Department of Psychology, Kwansei Gakuin University, Uegahara, Nishinomiya, Hyogo, Japan 662-8501 (e-mail: kazai@ kwansei.ac.jp). cades, and the other time-locked to the offset of saccades. The latency from the locked-point of the latter was almost invariant regardless of different saccade sizes, whereas that of the former increased in proportion to the saccade amplitude. Therefore, the lambda response is believed to be elicited by the afferent inflow beginning at the fixation pause, since visual suppression (e.g., Volkmann, 1986) occurs during saccades. Yagi's (1979) finding was confirmed by other studies (Billings, 1989; Marton \& Szirtes, 1982; Szirtes et al., 1982; Thickbroom et al., 1991; Yagi, 1981a).

The lambda response is affected by such physical properties of the stimulus as illuminance (Gaarder, Krauskopf, Graf, Kropfl, \& Armington, 1964; Marton \& Szirtes, 1982; Yagi, 1998), by the check size of the checkerboard pattern (Kazai \& Yagi, 1999), and also by psychological factors (Yagi, 1981b). The lambda response, therefore, is regarded as a type of so-called visual-evoked potentials (VEPs). Some studies have compared the lambda response (or the substitute in raw EEGs) with the flash-evoked response (Barlow \& Cigánek, 1969; Evans, 1953; Green, 1957; Perez-Borja et al., 1962; Scott et al., 1967), with the motion VEP (Ebersole \& Galambos, 1973; Lesèvre \& Rémond, 1973; Rémond \& Lesèvre, 1971; Riemslag, van der Heijde, \& van Dongen, 1987; Thickbroom et al., 1991), with the pattern-reversal VEP (Billings, 1989; Kurtzberg \& Vaughan, 1973; Morton et al., 1974; Scott, Moffett, \& Bickford, 1981), and with the pattern-onset VEP (Szirtes, Marton, \& Urban, 1988). In particular, the P100 component of pattern-reversal VEP is very similar to the lambda response (Billings, 1989; Scott et al., 1981). These studies suggest that the EFRP can be an index of visual information processing similar to VEP. Furthermore, EFRPs have the advantage of allowing participants to move their eyes, 
in contrast to the recording of so-called VEPs, for which eye movements must be restricted. Thus, if EFRPs are used as an index, a variety of visual tasks can be implemented that allow participants to move their eyes freely. These tasks might be more practical than the restricted task for usual VEP. Yagi, Imanishi, Konishi, Akashi, and Kanaya (1998), for example, succeeded in evaluating lighting systems with EFRP as an electrophysiologicalindex. There is also the possibility that the EFRP can shed new light on the nature of visual information processing with saccades.

As was mentioned above, many studies have implied some similarities between the EFRP and the VEP. There have been, however, few studies (Kurtzberg \& Vaughan, 1973, 1977; Perez-Borja et al., 1962) that have examined the neural generator of the lambda response (or the substitute in raw EEGs), whereas many studies have examined that of the VEP (e.g., Ikeda et al., 1998; Nakamura et al., 1997; Seki et al., 1996; Shigeto, Tobimatsu, Yamamoto, Kobayashi, \& Kato, 1998). It is necessary to investigate the neural generator of the lambda response, to enable it to be used as a well-established electrophysiological index. The purpose of the present study was to compare the lambda response and the P100 component of patternreversal VEP-especially, to compare their equivalent current dipoles.

\section{EXPERIMENT 1}

\section{Method}

\section{Participants}

Nine right-handed males, 22-32 years of age, participated in the experiment. Informed consent was obtained after the experimental procedures were explained. They had normal vision and no history of neurological disorders.

\section{Stimulus and Apparatus}

The stimuli were presented on a CRT display (refresh rate of $100 \mathrm{~Hz}$ ) that was placed at a distance of $45 \mathrm{~cm}$ from the eyes of the participants. The participants were seated in front of the CRT display in a sound-proof and shielded room, with their heads moderately fixed in a headrest. A black-and-white $\left(1 \mathrm{~cd} / \mathrm{m}^{2}\right.$ and $\left.20 \mathrm{~cd} / \mathrm{m}^{2}\right)$ checkerboard pattern was presented on the CRT screen. The stimulating field subtended $28^{\circ} \times 28^{\circ}$, and the check size was $40 \mathrm{~min}$.

For the lambda response recording, two green fixation targets $\left(13 \mathrm{~min}\right.$ in diameter and $12 \mathrm{~cd} / \mathrm{m}^{2}$ ) were also presented $3^{\circ}$ to the right and to the left from the center of the screen. The participant was asked to make 30 saccades between the two targets at a constant pace during a trial of $33 \mathrm{sec}$. The participant practiced executing saccades at this pace with a metronome before the experimental trials, but the pace was self-generated by the participant during the experimental trials.

For the pattern-reversal VEP recording, one green fixation point was presented at the center of the display. The pattern reversed at the rate of $0.9 \mathrm{~Hz}$ during a trial of $33 \mathrm{sec}$. The participant monocularly observed the stimulus, using the right eye. The participant underwent six trials for each brain potential recording.

\section{Recordings}

The EEG was recorded from 29 tin electrodes mounted in a cap (Electro-cap International, Inc.). The electrodes were placed according to the 10-20 system (Jasper, 1958), along with an additional 10 sites, as follows: Fpz, Oz, POz, PO3, PO4, PO7, PO8, Iz, C5, and C6 (American Electroencephalographic Society, 1991). All the electrodes were referenced to the left earlobe. A pair of electrodes was placed at the outer canthus of each eye for the horizontal EOG recording, and another pair of electrodes was placed infra- and supraorbitally to the left eye for the vertical EOG recording. A ground electrode was attached between Fpz and Fz. Electrode impedance was kept below $5 \mathrm{k} \Omega$.

All signals were amplified by a SynAmp (NueroScan, Inc.) with DC mode, and the low-pass filter was set at $200 \mathrm{~Hz}$. These signals were digitized on line at $1000 \mathrm{~Hz}$ and stored on an MO disk.

\section{Averaging EEGs}

All raw EEGs and EOGs were reviewed off line on a computer terminal before averaging. To average EEGs for EFRP, offset points of saccades were determined by moving a graphic cursor manually on the horizontal EOG tracing on the computer terminal. Two hundred epochs without artifacts between $512 \mathrm{msec}$ before and $512 \mathrm{msec}$ after the offset point of the saccade were averaged for EFRP. The direction of the eye movement was counterbalanced within each participant. The number of epochs for averaged VEP was more than 200 . Both potentials were digitally low-pass filtered at $50 \mathrm{~Hz}$ after averaging.

\section{Dipole Tracing}

We applied the dipole tracing method (Musha \& Homma, 1990) to calculate equivalent current dipoles, which uses a real shape head model of a uniform conductor (Musha \& Homma, 1990; Scherg \& Von Cramon, 1985). The head shape of the participant and the location of the electrode were digitized using a three-dimensional digitizer (3 SPACE, ISOTRAK II). The head coordinate system was employed for the dipole localization. In the coordinate system, $x$ (anterior), $y$ (lateral), and $z$ (superior) axes were defined using the nasion and preauricular points as fiducial points. The origin was the point halfway between the preauricular points. The calculation was based on one- or two-dipole models. Calculated dipoles were treated as meaningful when the dipolarity exceeded $95 \%$ in the one-dipole model or $97 \%$ in the two-dipole model. Dipolarity was defined as the square root of 1.0 minus the sum of the squares of the difference between the measured potential and the calculated potential, normalized by the sum of the squares of the measured potential (Musha \& Homma, 1990).

\section{Results}

\section{Averaged Potentials}

Separate waveforms for pattern-reversal VEP and EFRP of the 9 participants are shown in Figure 1. The baselines are computed as the mean of all data points from 100 to $81 \mathrm{msec}$ prior to the offset point of the saccade for EFRP and from 20 to $1 \mathrm{msec}$ prior to the onset of the pattern reversal for pattern-reversal VEP.

The P100 component of pattern-reversal VEP was dominant at the occipital site. The lambda response of the EFRP was also dominant at the occipital site. They, however, appeared to be morphologically different: The peak latency of the lambda response at $\mathrm{Oz}[M=82.8 \mathrm{msec}$, $S D=3.42 \mathrm{msec}]$ was shorter than that of the P100 $[M=$ $106.9 \mathrm{msec}, S D=3.76 \mathrm{msec} ; t(8)=19.79, p<.01$, twotailed]. This finding of a difference in the latencies agrees with a previous study that used the offset of saccades as the trigger to average EEGs for the lambda response (Billings, 1989). On the other hand, there was no significant difference in the amplitude between the lambda response $(M=7.27 \mu \mathrm{V}, S D=2.89 \mu \mathrm{V})$ and the $\mathrm{P} 100[M=$ $7.72 \mu \mathrm{V}, S D=5.27 \mu \mathrm{V} ; t(8)=0.44, p=.67]$. This result did not agree with the previous study (Billings, 1989), 

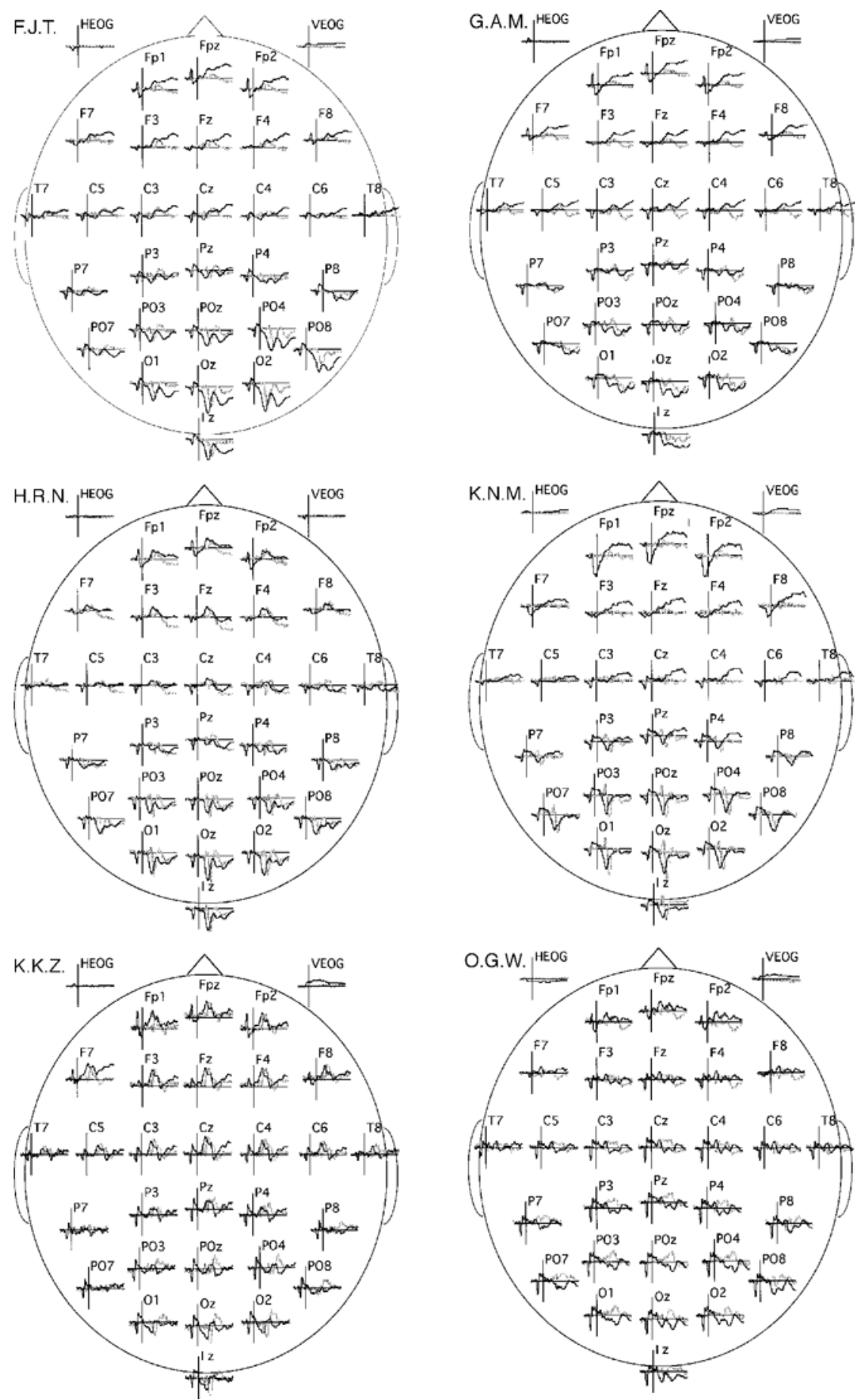

Figure 1. Averaged waveforms of EFRP and pattern-reversal VEP for the 9 participants. Note that the origins indicate the offset of saccade for the EFRP and the onset of pattern reversal for pattern reversal $\mathrm{VEP}$.

which reported that the amplitude of the lambda response was larger than that of the P100. This discrepancy might be due to the difference in the stimulus parameters between the studies.

\section{Dipole Tracing}

Equivalent current dipoles were estimated at five consecutive time points around the peak of the most prominent component at $\mathrm{Oz}$ with an interval of $1 \mathrm{msec}$. Both the 

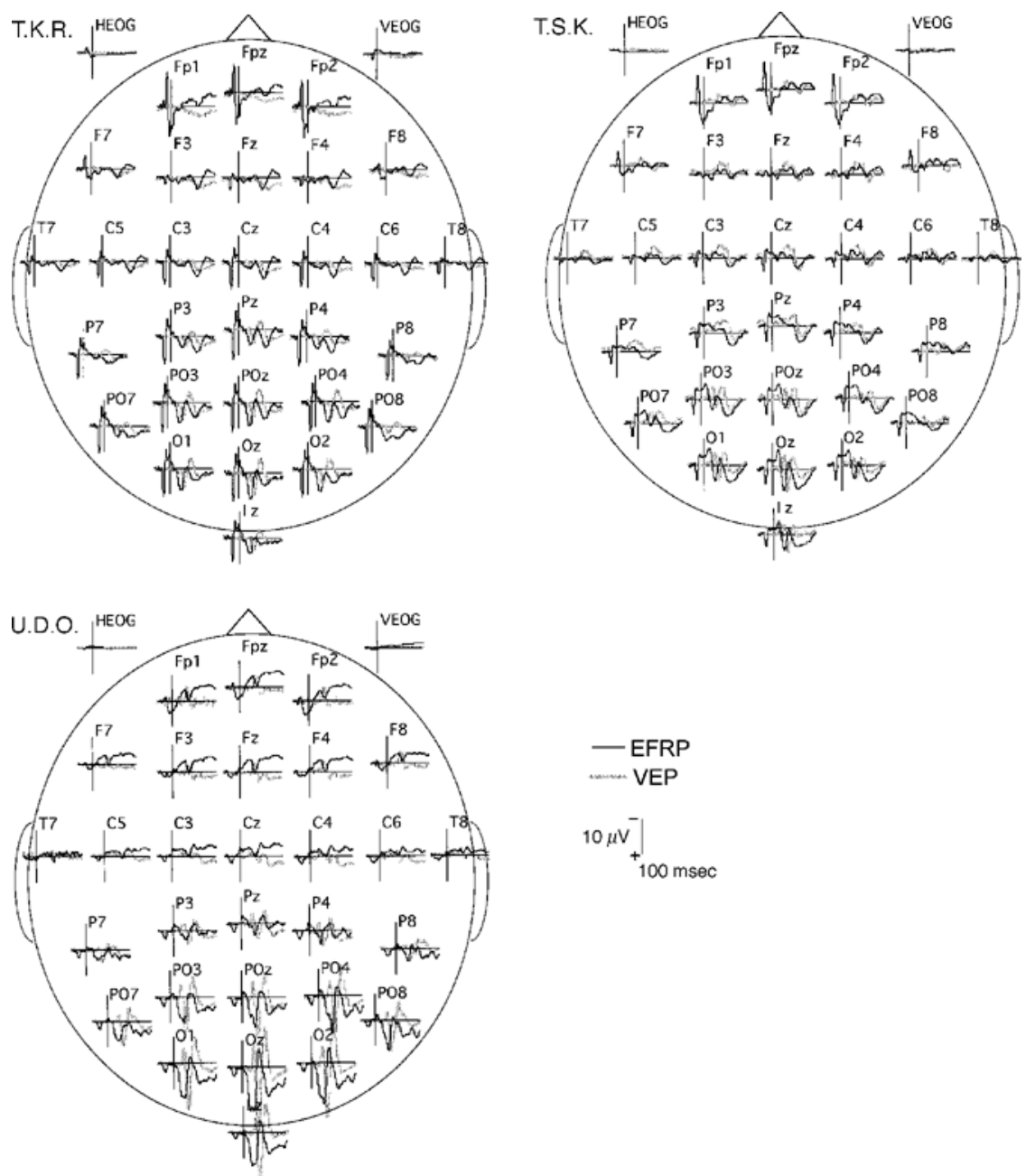

Figure 1. (Continued).

one-dipole model and the two-dipole model were used to calculate the dipoles, and one of them was adopted on the basis of the following criterion. Dipoles with a dipolarity exceeding $95 \%$ were evaluated as equivalent current dipoles for the one-dipole model, and $97 \%$ was used for the two-dipole model. Furthermore, the estimated dipole models were rejected unless the dipolarities exceeded the criterion at all the five consecutive points. The EEG data were first subjected to the one-dipole estimation and then to the two-dipole estimation if the one-dipole model was rejected. All 29 electrode sites were used to calculate the dipoles.

Figure 2 shows the locations of dipoles estimated by the dipole-tracing method at the peaks of the lambda response and P100. Squares indicate the estimated dipoles for the lambda response, and circles for the P100. In all the participants, the one-dipole model met the criteria for the lambda response and for the P100. The dipoles of the P100 were located at the occipital site for all the participants. The dipoles of the lambda response were also located at the occipital site very close to those of the P100. Paired $t$ tests (two-tailed) were used to test the difference of the dipole locations between the lambda response and the P100 for each axis. In the $x$-axis, the dipole location of the lambda response $(M=-28 \mathrm{~mm}, S D=12 \mathrm{~mm})$ was marginally more anterior to that of the P100 $[M=-34 \mathrm{~mm}$, $S D=8 \mathrm{~mm} ; t(8)=1.82, p=.11]$. In the $y$-axis and the $z$-axis, the dipole locations of the lambda response $(M=5 \mathrm{~mm}$, $S D=6 \mathrm{~mm}$, and $M=63 \mathrm{~mm}, S D=7 \mathrm{~mm}$, respectively) were not significantly different from those of the P100 [M= $5 \mathrm{~mm}, S D=9 \mathrm{~mm}$, and $M=66 \mathrm{~mm}, S D=8 \mathrm{~mm} ; t(8)=$ $0.05, p=.96$, and $t(8)=0.93, p=.38$, respectively]. These results suggested that the lambda response might include activities of other parts (cortical or extracortical) that are not included in the P100 (discussed below).

To address more clearly about the location of the generator of the lambda response, half-visual-field stimulation and patternless stimulation were employed in Experiment 2.

\section{EXPERIMENT 2}

The aim of the present study was to provide evidence for the use of the lambda response as an electrophysiological index of visual information processing, instead of other VEP. Therefore, for this purpose, it would be reason- 

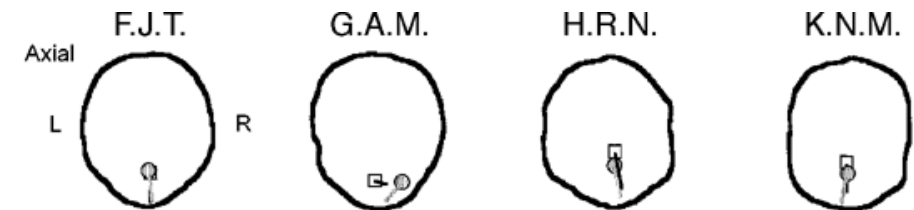

K.K.Z.
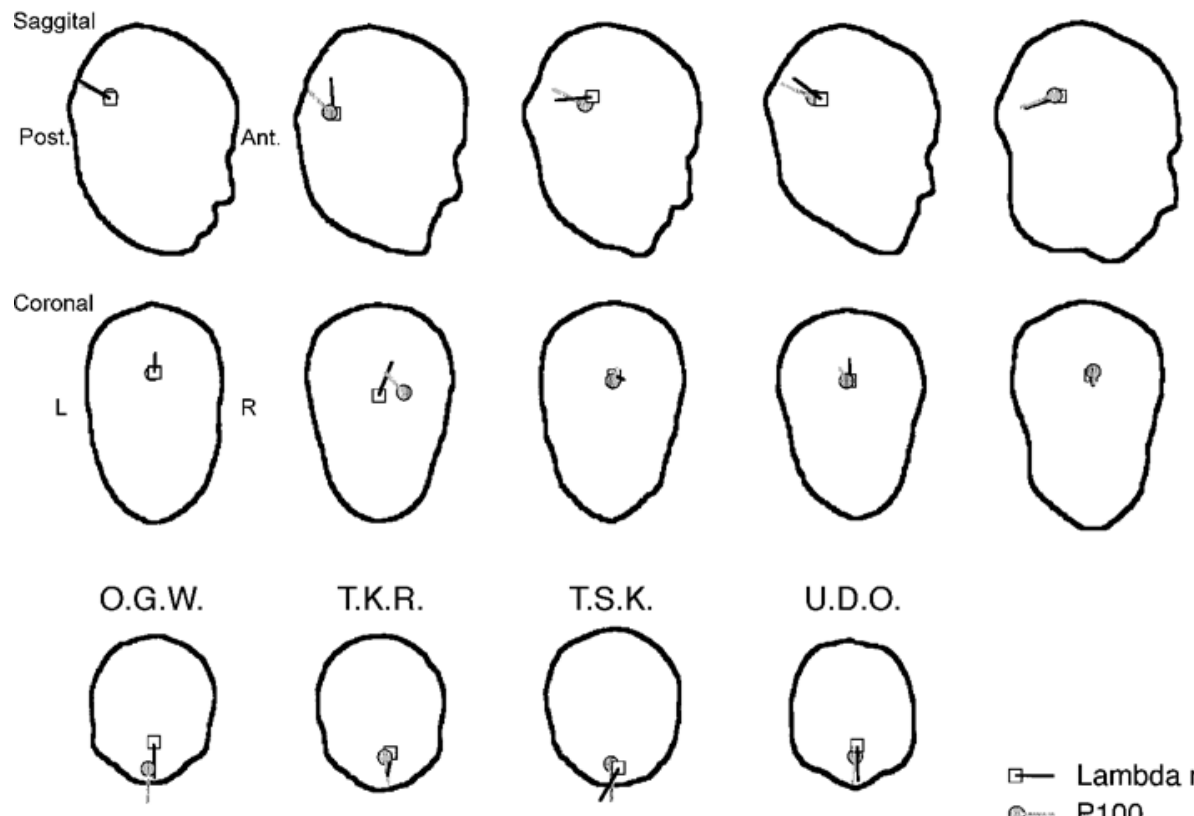

T.K.R.
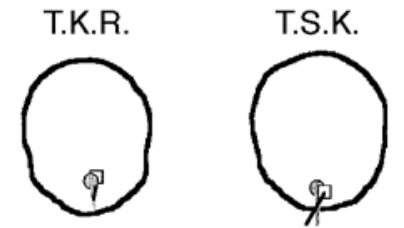

U.D.O.
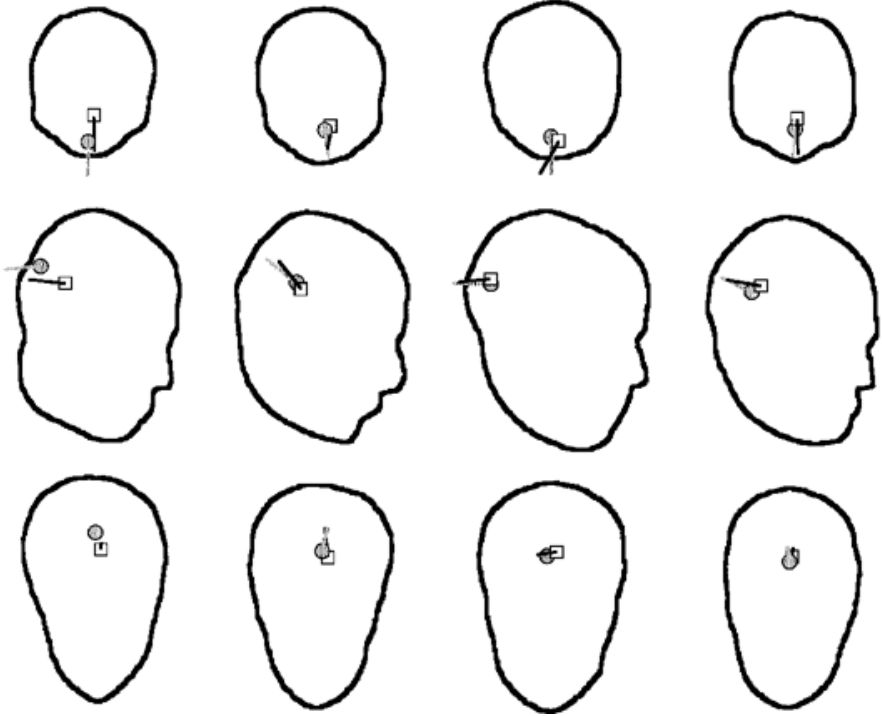

Figure 2. Results of dipole estimation at the peaks of the lambda response and the P100. Squares indicate the estimated dipoles for the lambda response, and circles indicate those for the P100 component.

able to focus on the activities evoked by visual stimulation. As is already known, the execution of saccadic eye movements in itself accompanies electrical activities (e.g., Evinger et al., 1994; Gaymard, Ploner, Rivaud, Vermersch, \& Pierrot-Deseilligny, 1998; Pierrot-Deseilligny, Gaymard, Müri, \& Rivaud, 1997). This finding implies that EFRP involves additional bioelectrical activities, because the recording of EFRP requires saccadic eye movements, and that those activities would have affected dipole localization in Experiment 1. Thus, in Experiment 2, EFRPs without stimulus patterns were recorded in order to evaluate those activities. Furthermore, EFRP and pattern-reversal VEP to half-field stimuli were also recorded in order to compare more precisely the generator of the lambda response with that of the P100 component. It is already known that the P100 to the vertical half-field stimulus is dominant at the ipsilateral occipital electrode (e.g., Barrett, Blumhardt, Halliday, Halliday, \& Kriss, 1976) and that the dipole of the P100 is estimated in the contralateral hemisphere with its direction toward the medial (Nakamura et al., 1997; Seki et al., 1996; Shigeto et al., 1998).

\section{Method}

The method was the same as that in Experiment 1, except for the participants and the stimulus.

\section{Participants}

Seven right-handed males, 22-32 years of age, participated in the experiment. Six of the participants had participated in Experiment 1. 
Informed consent was obtained after the experimental procedures were explained. They had normal vision and no history of neurological disorders.

\section{Stimulus}

A black-and-white $\left(1 \mathrm{~cd} / \mathrm{m}^{2}\right.$ and $\left.20 \mathrm{~cd} / \mathrm{m}^{2}\right)$ checkerboard pattern was presented on the CRT screen. The stimulating field subtended $42^{\circ}$ horizontally and $32^{\circ}$ vertically, and the check size was $60 \mathrm{~min}$. For the lambda response recording, two green fixation targets (13 min in diameter and $12 \mathrm{~cd} / \mathrm{m}^{2}$ ) were presented $3^{\circ}$ above and below the center of the screen; thus, the participants were asked to make saccades vertically. For the pattern-reversal VEP recording, one green fixation point was presented at the center of the screen. The half-field stimulus pattern $\left(18^{\circ} \times 32^{\circ}\right)$ was on the black background, and the inner edge of the pattern was $3^{\circ}$ lateral to the fixation point(s). No stimulus pattern other than the two fixation points was presented in the no-stimulus condition for the lambda response recording.

\section{Results}

\section{Averaged Potentials}

Figure 3 shows separate waveforms for pattern-reversal VEP and EFRP at occipital sites of the 7 participants. It should be noted that EFRPs were evoked even in the nostimulus condition, in which there was no stimulus pattern (Figure 3A). This result indicates that the saccade by itself yielded potentials associated with the offset of the saccade. Difference waveforms were calculated by subtracting the waveform in the no-stimulus condition from the waveform in the other conditions (Figure 3B).

The peak latencies and amplitudes at $\mathrm{Oz}$ to the fullfield stimulus were submitted to repeated measures analyses of variance (ANOVAs), and least significant difference (LSD) tests were used for the multiple comparison. As for the peak latency, the main effect was significant $\left[F(2,12)=168, M S_{\mathrm{e}}=10.72, p<.01\right]$. LSD tests revealed that the peak latencies of the lambda response $(M=$ $80.7 \mathrm{msec}, S D=4.9 \mathrm{msec}$ ) and the subtracted lambda response $(M=78.4 \mathrm{msec}, S D=5.0 \mathrm{msec})$ were significantly shorter than that of the P100 $(M=107.3 \mathrm{msec}, S D=$ $3.0 \mathrm{msec}$; both $p \mathrm{~s}<.01$ ); however, there was no significant difference between the lambda response and the subtracted lambda response $(p=.22)$. For the peak amplitude, the main effect was not significant $[F(2,12)=0.75$, $\left.M S_{\mathrm{e}}=5.03, p=.49\right]$, and there was no significant difference between the lambda response $(M=9.85 \mu \mathrm{V}, S D=$ $2.84 \mu \mathrm{V})$, the subtracted lambda response $(M=10.14 \mu \mathrm{V}$, $S D=2.49 \mu \mathrm{V})$ and the $\mathrm{P} 100(M=8.75 \mu \mathrm{V}, S D=2.60 \mu \mathrm{V}$; the least probability of Type I error was .27 among all the comparisons). These results agree with those in Experiment 1 . These results also indicate that the subtraction did not distort the lambda response, especially in the temporal property.

For the half-field stimuli, the paradoxical lateralization (Barrett et al., 1976) was clearly observed on the P100: The half-field stimuli evoked larger responses in the ipsilateral sites than in the contralateral sites (Figure 3C). The lambda response also showed the paradoxical lateralization, although it was less discernible than that for the P100 (Figure 3A). It became slightly clearer in the difference waveforms that were yielded by subtracting the waveform in the no-stimulus condition from the waveforms in the half-field stimulus conditions (Figure 3B).

\section{Dipole Tracing}

The criterion and procedure for dipole estimation were the same as those in Experiment 1.

Full-field stimulus. First, the equivalent current dipoles of the lambda response and the P100 to the full-field stimulus were estimated. The one-dipole model was applied to the P100 in all the participants and to the lambda response in 6 participants, and all of these dipoles were located at the occipital site. The two-dipole model was applied to the lambda response in participant T.S.K., with the first dipole located at the occipital site and the second at the frontal site. The dipoles of the lambda response in the nostimulus condition were also estimated, but no trend in their locations was observed between the participants. Then the dipoles of the subtracted lambda response were estimated. For the subtracted lambda response, the onedipole model was applied to all the participants, and all of the dipoles were located at the occipital site. The dipole locations, except those in the no-stimulus condition, were submitted to repeated ANOVAs for each axis, and LSD tests were used for multiple comparisons.

On the $x$-axis, where the main effect was significant $\left[F(2,12)=4.55, M S_{\mathrm{e}}=57.45, p=.03\right]$, the dipole location of the lambda response $(M=-24 \mathrm{~mm}, S D=14 \mathrm{~mm})$ was significantly more anterior both to that of the P100 $(M=$ $-35.93 \mathrm{~mm}, S D=10 \mathrm{~mm})$ and to that of the subtracted lambda response $(M=-33.16 \mathrm{~mm}, S D=11.75 \mathrm{~mm} ; p \mathrm{~s}=$ .01 and .04 , respectively). On the other hand, the dipole location of the subtracted lambda response was not significantly different from that of the P100 $(p=.54)$. On the $y$ axis, the main effect was not significant $[F(2,12)=0.29$, $\left.M S_{\mathrm{e}}=4.19, p=.75\right]$. Also, on the $z$-axis, the main effect was not significant $\left[F(2,12)=0.44, M S_{\mathrm{e}}=59.39, p=.65\right]$.

These results support the idea that the lambda response shares a common neural generator with the P100 and involves other kinds of bioelectrical activities than the activities evoked by the visual stimulus (discussed below). We can, furthermore, assume that the same activities affected the dipole localization in Experiment 1; consequently, the dipole of the lambda response was located more anterior to that of the P100.

Half-field stimulus. For the half-field stimuli, we focused on the comparison of the equivalent current dipoles of the subtracted lambda responses with those of the P100 because the dipole localization for the full-field stimulus suggested that the subtracted lambda response was more appropriate for the comparison. However, the point at which the dipole was estimated corresponded to the peak latency of the most prominent component at $\mathrm{Oz}$ in the full-field stimulated response, which was considered to be the neutral point for both of the half-field (left/right) stimulations.

The two-dipole model was applied to 4 participants (F.J.T., K.K.Z., M.S.K., and T.K.R.) for the subtracted lambda response to the right-half-field stimulus and was also applied to 1 participant (T.K.R.) for that to the left- 
F.J.T.
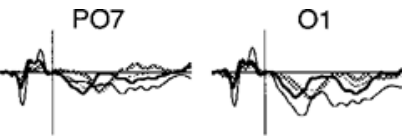

$4^{\mathrm{Oz}}$
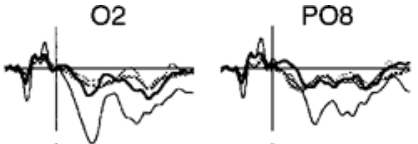

(B)
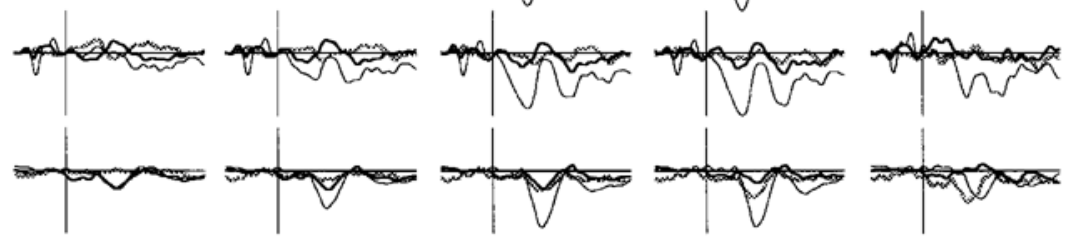

(C)

H.R.N
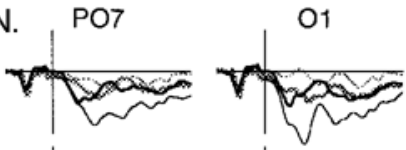

(B)
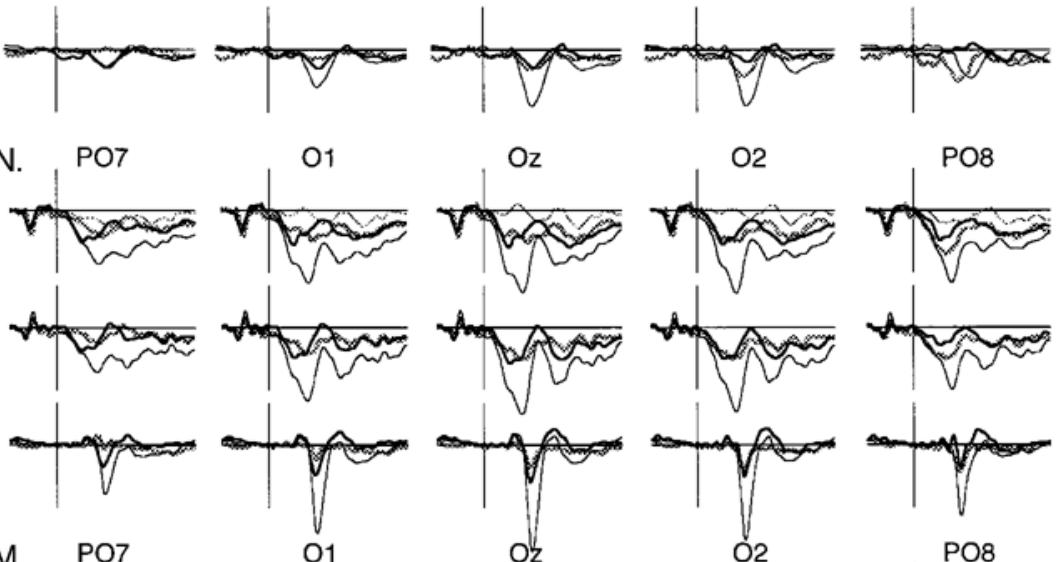

(C)

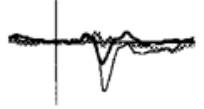

01

(A)
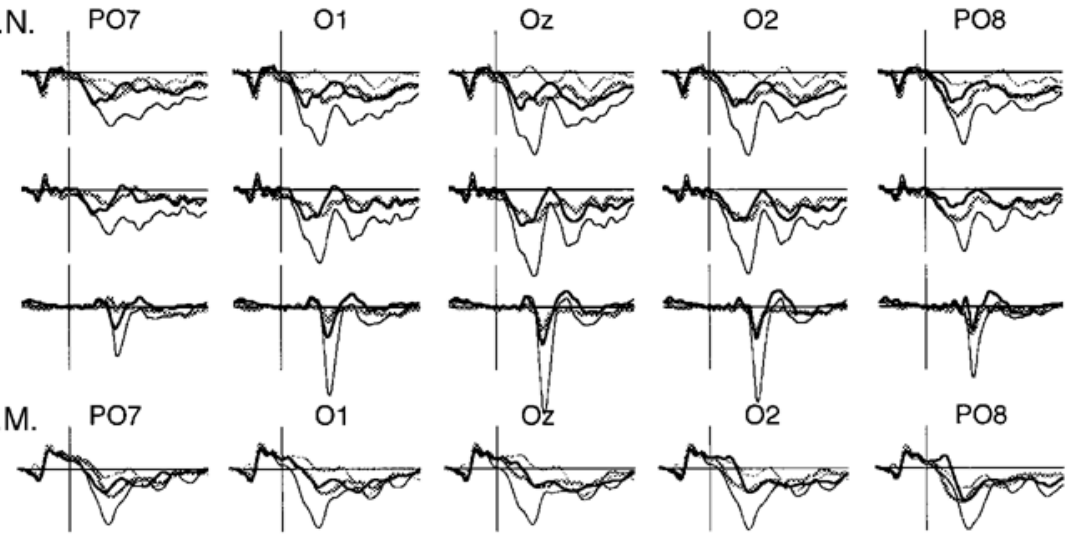

(B)
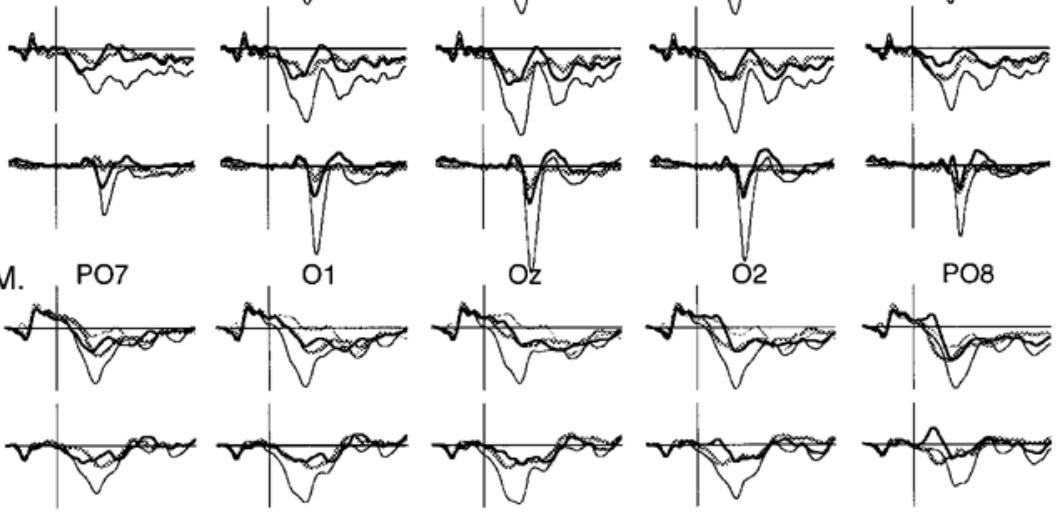

(C)
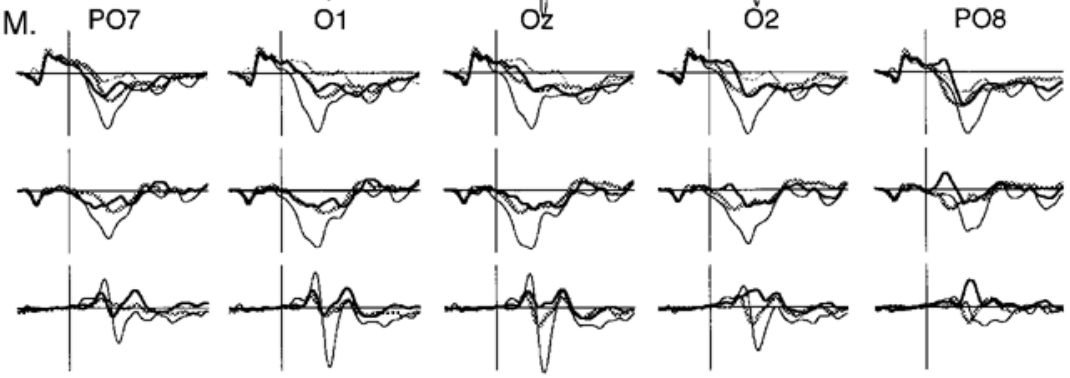

K.K.Z.
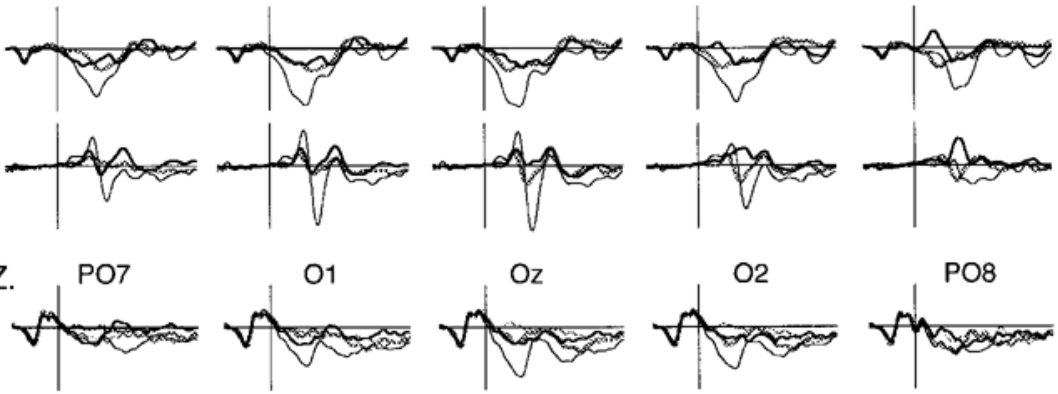

(B)
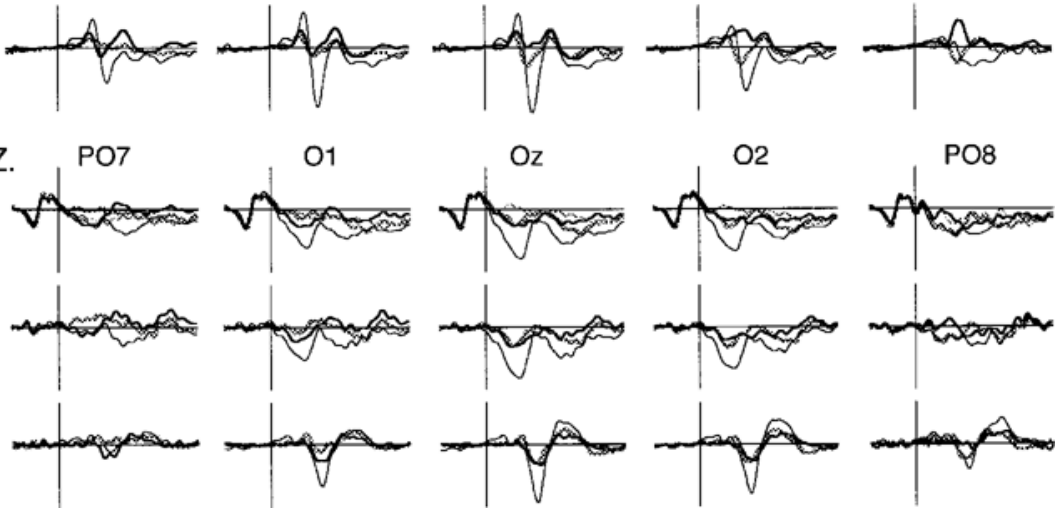

(C)
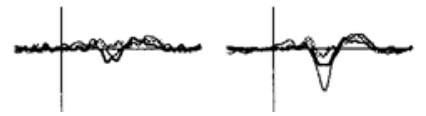

Figure 3. Averaged waveforms for pattern-reversal VEP and EFRP at occipital sites in the 7 participants. (A) Original EFRP of all conditions. (B) The difference waveforms that were yielded by subtracting the waveform of the no-stimulus condition from the waveforms of the other conditions. (C) Pattern-reversal VEP of all conditions.

half-field stimulus. In these cases, no trend in the second dipole between participants or within participant was found, and all the first dipoles were estimated at the occipital site. Therefore, the application of the two-dipole model is probably due to the lower amplitude of the response or the lower signal-to-noise ratio (Figure 3B). For this reason, the second dipoles were excluded from further analysis in the present study. In the other cases, the one-dipole model was applied, and all of the dipoles were estimated at the occipital site. For the P100, the onedipole model was applied in all cases, and the dipoles were estimated in the occipital site.
Figure 4 shows the locations of the occipital dipoles to the half-field stimuli. For both the subtracted lambda response and the P100, the dipoles were estimated in the contralateral sides at the occipital site, with their directions toward the medial. Table 1 indicates the mean locations of the dipoles across the participants for each axis. These dipole locations were submitted to repeated measures $2 \times 2($ left/right $\times$ lambda/P100) ANOVAs for each axis.

On the $x$-axis, none of the main effects was significant [left/right, $F(1,6)=0.04, M S_{\mathrm{e}}=116.29, p=.85$; lambda/ $\left.\mathrm{P} 100, F(1,6)=1.27, M S_{\mathrm{e}}=93.42, p=.30\right]$. The interaction 


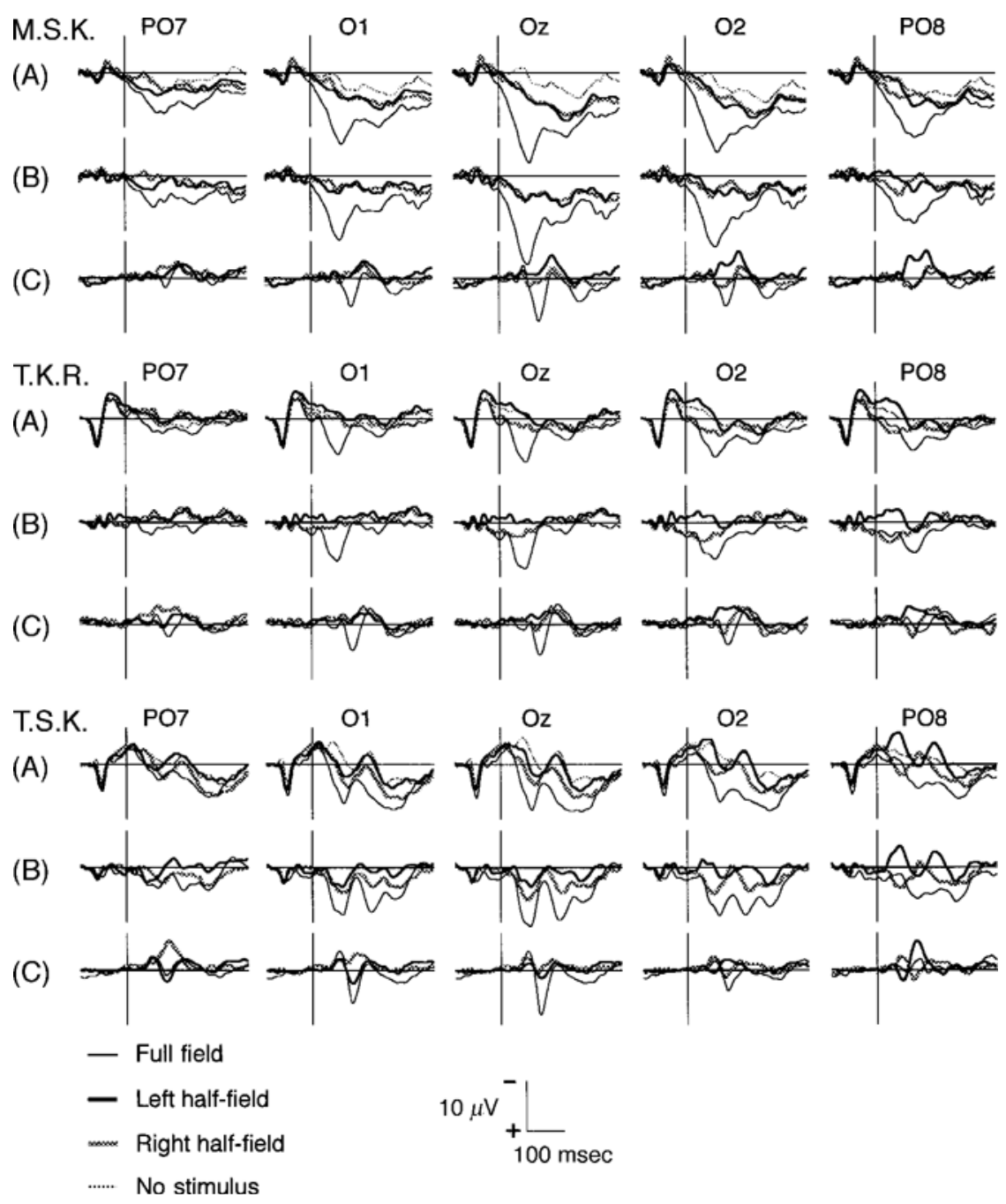

Figure 3. (Continued).

between left/right and lambda/P100 was marginally significant $\left[F(1,6)=3.14, M S_{\mathrm{e}}=55.16, p=.13\right]$; however, multiple comparisons using LSD tests did not reveal any significant differences (the least probability of Type I error was .20 among all the comparisons). On the $y$-axis, the main effect of left/right was significant $[F(1,6)=$ $\left.125.17, M S_{\mathrm{e}}=24.07, p<.01\right]$, whereas the main effect of lambda/P100 and the interaction were not $[F(1,6)=0.01$, $M S_{\mathrm{e}}=62.88, p=.91$, and $F(1,6)=0.00, M S_{\mathrm{e}}=73.05, p=$ .98 , respectively]. On the $z$-axis, the main effects and the interaction were not significant [left/right, $F(1,6)=0.21$, $M S_{\mathrm{e}}=49.98, p=.66 ;$ lambda $/ \mathrm{P} 100, F(1,6)=0.17, M S_{\mathrm{e}}=$ $179.52, p=.69 ;$ left $/$ right $\times$ lambda/P100, $F(1,6)=0.19$, $\left.M S_{\mathrm{e}}=67.57, p=.68\right]$. All of these results amount to indicating that the dipoles were estimated in the contralateral sides when half-field stimuli were used and that there was no significant difference between the dipole location of the subtracted lambda response and that of the P100.

\section{GENERAL DISCUSSION}

The dipoles of the P100 and the lambda response at the occipital site were estimated at approximately the same location, especially when the subtracted lambda response was used to estimate its dipole (Figures 2 and 4). Recent studies have indicated that the neural generator of the P100 lies around the calcarine fissure (Nakamura et al., 1997; Seki et al., 1996; Shigeto et al., 1998). Therefore, the present finding suggests that the lambda response also has its neural generator around the calcarine fissure. This suggestion coincides with previous studies with direct recordings from the cortex (Perez-Borja et al., 1962; Salzarulo, Lairy, \& Bancaud, 1976). However, it should be noted that the dipole of the lambda response without the subtraction was located in a more anterior part than that of the P100. The difference was significant in Experiment 2 and marginally significant in Experiment 1 . As 
F.J.T.

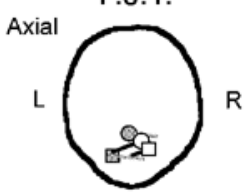

H.R.N.
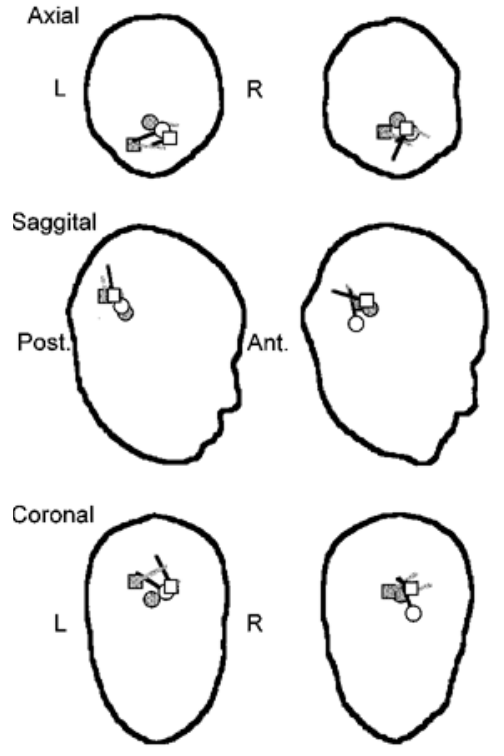

T.K.R.
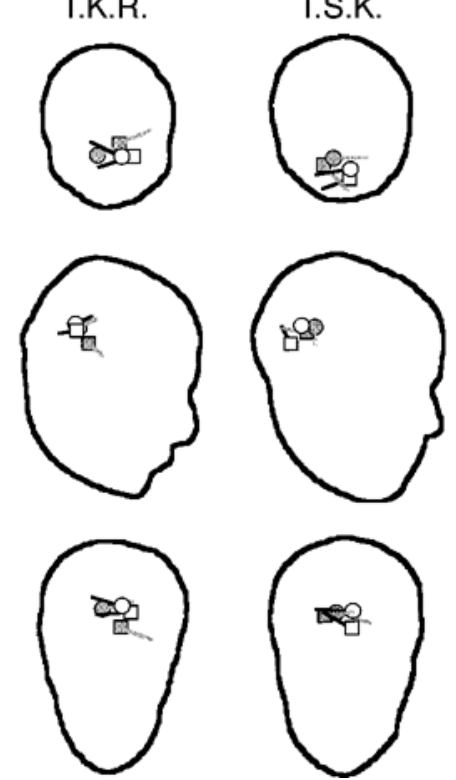

K.N.M.

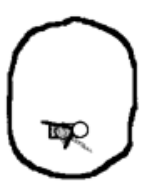

K.K.Z.

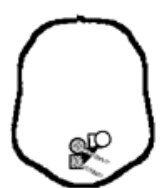

M.S.K.
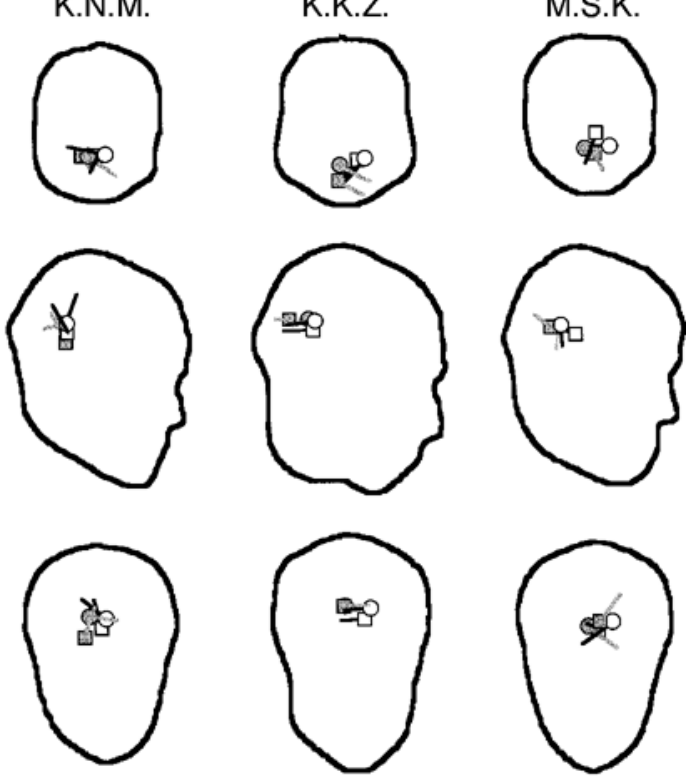

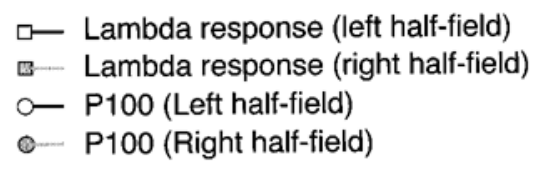

口- Lambda response (left half-field)

[-- Lambda response (right half-field)

-.- P100 (Right half-field)

Figure 4. Results of the dipole estimation of the subtracted lambda response and the P100. Gray squares indicate the dipoles of the lambda response to the right-half-field stimulus, and open squares indicate those to the left-half-field stimulus. Gray circles indicate the dipoles of the P100 to the right-half-field stimulus, and open circles indicate those to the left-half-field stimulus. Note that the dipoles of the lambda response were estimated after the subtraction. The two-dipole model was applied to 4 participants (F.J.T., K.K.Z., M.S.K., and T.K.R.) for the subtracted lambda response to the right-half stimulation and was also applied to 1 participant (T.K.R.) for that to the lefthalf stimulation. These second dipoles, however, are not plotted here, because they had no trend between participants or within participant, and they were excluded from further analysis.

was mentioned above, the difference is probably due to additional bioelectrical activities (cortical or extracortical) that are involved in the lambda response. It is reasonable to think that the contribution from these additional activities to the dipole localization was eliminated by subtract- ing the EFRP in the no-stimulus condition from the EFRP in the other conditions.

It is well known, for example, that saccadic eye movements accompany cortical activities other than those in the occipital lobe. This means that the execution of the sac- 
Table 1

Locations of the Dipoles of the Subtracted Lambda Response and P100

\begin{tabular}{lrrrrrrrr}
\hline & \multicolumn{3}{c}{ Left Half-Field } & & \multicolumn{3}{c}{ Right Half-Field } \\
\cline { 2 - 3 } \cline { 7 - 8 } & $x$-axis & $y$-axis & $z$-axis & & $x$-axis & $y$-axis & $z$-axis \\
\hline Lambda response & & & & & & & \\
$M$ & -24.3 & 15.3 & 60.7 & & -30.0 & -5.5 & 63.3 \\
$S D$ & 13.3 & 9.7 & 10.2 & & 17.7 & 14.2 & 12.3 \\
P100 & & & & & & \\
$M$ & -25.1 & 15.6 & 64.2 & & -20.9 & -5.1 & 64.1 \\
$S D$ & 6.4 & 8.0 & 11.7 & & 9.8 & 5.6 & 6.6 \\
\hline
\end{tabular}

Note-Locations are given in millimeters. Positive values indicate anterior on the $x$-axis, leftward on the $y$-axis, and upward on the $z$-axis. $N=7$.

cade itself accompanies activities of particular cortical areas that have no direct relation to visual sensory input. At least three different cortical areas are considered to be involved in the execution of the saccade in a task such as that in the present experiments: the parietal eye field, the frontal eye field (FEF), and the supplementary eye field (for reviews see Gaymard et al., 1998; Pierrot-Deseilligny et al., 1997). These additional cortical activities might have affected the dipole localization of the lambda response. However, it is less likely that the contribution from these activities to the dipole localization was very large, because these cortical areas are thought to be engaged in both preparing and triggering saccades. For example, most activities in the FEF, whose neurons project to the superior colliculus, are terminated by the completion of saccades (Bruce \& Goldberg, 1985). In contrast, the lambda response is considered to be evoked after the offset of saccades (Yagi, 1979). Indeed, the dipoles for the lambda response were calculated at the peak latency of the lambda response-that is, at some $80 \mathrm{msec}$ after the offset of saccades. Thus, the interval between the activation of these areas and the peak latency of the lambda response is very large.

It is more likely that the primary visual cortex activity subordinate to the saccade affected the dipole localization. There is evidence that indicates activation in the primary visual cortex by the saccade itself (Bodis-Wollner et al., 1997). Bodis-Wollner et al. investigated the activated areas during saccades, using functional magnetic resonance imaging. They found that voluntary saccades activated the primary visual cortex even in the dark. As for the timing of the activation, Park and Lee (2000) showed that the neural activity in the cat's area 17 and area 18 increased in relation to the offset of a saccade even in the case with no visual stimulus. It seems that this activation extends to the anterior part of the calcarine fissure, where more peripheral visual field is represented than the visual field stimulated in the present experiments (Bodis-Wollner et al., 1997, Figure 3). Therefore, because this part could not be activated in the VEP trials of the present experiments, this activation might have shifted the dipole of the lambda response toward the anterior part to that of the P100.

There is another possibility that extracortical activities were involved in the lambda response-namely, that is, the potential change induced by eyelid movement subor- dinate to saccades affected the dipole localization for the lambda response. Evinger et al. (1994) reported that not only vertical eye movements, but also horizontal saccades could accompany eyelid movements. In the present study, the most typical example of the eyelid movement potential was observed in Participant T.S.K. in Experiment 2. The two-dipole model was applied to his lambda response without the subtraction in the half-field conditions, as well as in the full-field condition. For this participant, the second dipoles were estimated at the frontal site, and the vertical EOG showed a slight deflection of some $2 \mu \mathrm{V}$ at the point at which the dipoles were estimated. The location of the second dipole indicates that the eyelid movement potential of Participant T.S.K. changed the electrical distribution on his scalp, to yield the second dipole. It is likely that the influence of the eyelid movement potential on the dipole localization of the lambda response has a large interindividual difference, since the amplitude of the deflection in the vertical EOG had a large interindividual difference, ranging from more than $2.0 \mu \mathrm{V}$ to less than $0.2 \mu \mathrm{V}$, in the present study. Therefore, the eyelid movement did not necessarily yield the second dipole at the frontal site but shifted the location of the first dipole toward the frontal site. It should be noted that this kind of eyelid movement is not the so-called eye blink, because the eye blinks of the participants in the present study generated deflections of more than $400 \mu \mathrm{V}$ in the vertical EOG; the epochs that showed such a large deflection were excluded from the averaging.

As was mentioned above, the present results suggest that the lambda response and the P100 arise from common neural generators. Nevertheless, there is a certain difference in the latency between the lambda response and the P100: The peak latency of the lambda response was $24 \mathrm{msec}$ shorter than that of the P100 in Experiment 1 and was $27 \mathrm{msec}$ shorter in Experiment 2. It is known that both the lambda response and the P100 depend on various aspects of the stimulus (e.g., Kazai \& Yagi, 1999; KuritaTashima, Tobimatsu, Nakayama-Hiromatsu, \& Kato, 1991), but the aspects of the stimulus used in the present experiments were the same between the VEP trials and the EFRP trials. Thus, the difference in the latency could not be attributed to aspects of the stimulus. The crucial point was that the saccadic eye movement was required for recording the lambda response but the fixation was required for recording the $\mathrm{P} 100$. Then, the difference in the peak latency also could be explained by the activation in the primary visual cortex subordinate to saccades (Bodis-Wollner et al., 1997; Park \& Lee, 2000). This cortical activation might facilitate the activity caused by the visual stimulus in the present experiment and might yield the differences in the latencies between the lambda response and the P100. Provided that the peak of the lambda response reflects the same visual information processing as that of the $\mathrm{P} 100$, the visual information processing at the fixation following the saccade may begin prior to the end of the saccade, or the saccade may facilitate information processing after the saccade, to compensate for the visual inefficiency caused by the saccadic suppression. 


\section{REFERENCES}

American Electroencephalographic Society (1991). American Electroencephalographic Society guidelines for standard electrode position nomenclature. Journal of Clinical Neurophysiology, 8, 200-202.

BARlow, J. S., \& CigáneK, L. (1969). Lambda responses in relation to visual evoked responses in man. Electroencephalography \& Clinical Neurophysiology, 26, 183-192.

Barret, G., Blumhardt, T., Halliday, A. M., Halliday, E. \& Kriss, A. (1976). A paradox in the lateralization of the visual evoked response. Nature, 261, 253-255.

Billings, R. J. (1989). The origin of the occipital lambda wave in man. Electroencephalography \& Clinical Neurophysiology, 72, 95-113.

Bodis-Wollner, I., Bucher, S. F., Seelos, K. C., Paulus, W., ReISER, M., \& OERTEL, W. H. (1997). Functional MRI mapping of occipital and frontal cortical activity during voluntary and imagined saccades. Neurology, 49, 416-420.

Bruce, C. J., \& Goldberg, M. E. (1985). Primate frontal eye fields: I. Single neurons discharging before saccades. Journal of Neurophysiology, 53, 603-635.

Ebersole, J. S., \& Galambos, R. (1973). Lambda waves evoked by retinal stimulation in the absence of eye movement. Electroencephalography \& Clinical Neurophysiology, 35, 39-47.

Evans, C. C. (1953). Spontaneous excitation of the visual cortex and association areas-lambda waves. Electroencephalography \& Clinical Neurophysiology, 5, 69-74.

Evinger, C., Manning, K. A., Pellegrini, J. J., Basso, M. A., PowERS, A. S., \& Sibony, P. A. (1994). Not looking while leaping: The linkage of blinking and saccadic gaze shifts. Experimental Brain Research, 100, 337-344.

GaArder, K., Krauskopf, J., Graf, V., Kropfl, W., \& Armington, J. C. (1964). Averaged brain activity following saccadic eye movement. Science, 146, 1481-1483.

Gaymard, B., Ploner, C. J., Rivaud, S., Vermersch, A. I., \& PierrotDeseilligny, C. (1998). Cortical control of saccades. Experimental Brain Research, 123, 159-163.

GreEN, J. (1957). Some observations on lambda waves and peripheral stimulation. Electroencephalography \& Clinical Neurophysiology, 9 , 691-704.

Ikeda, H., Nishijo, H., Miyamoto, K., Tamura, R., Endo, S., \& Ono, T. (1998). Generators of visual evoked potentials investigated by dipole tracing in the human occipital cortex. Neuroscience, 84, 723-739.

JASPER, H. H. (1958). The ten-twenty electrode system of the International Federation. Electroencephalography \& Clinical Neurophysiology, 10, 371-375.

KAZAI, K., \& YAGI, A. (1999). Integrated effect of stimulation at fixation points on EFRP (eye-fixation-related brain potentials). International Journal of Psychophysiology, 32, 193-203.

Kurita-Tashima, S., Tobimatsu, S., Nakayama-Hiromatsu, M., \& KATO, M. (1991). Effect of check size on the pattern reversal visual evoked potential. Electroencephalography \& Clinical Neurophysiology, 80, 161-166.

Kurtzberg, D., \& VAughan, H. G. (1973). Electrocortical potentials associated with eye movements. In V. Zikmund (Ed.), The oculomotor system and brain functions (pp. 137-146). London: Butterworths.

Kurtzberg, D., \& Vaughan, H. G. (1977). Electrophysiological observations on the visuomotor system and visual neurosensorium. In J. E. Desmedt (Ed.), Visualevoked potentials in man: New developments (pp. 314-331). Oxford: Oxford University Press, Clarendon Press.

Lesèvre, N., \& RÉmond, A. (1973). Effects of contrast on the visual evoked potentials related to eye movements. In V. Zikmund (Ed.), The oculomotor system and brain function (pp. 119-134). London: Butterworths.

Marton, M., \& Szirtes, J. (1982). Averaged lambda potential and visual information processing. Studia Psychologica, 24, 165-170.

Morton, H. B., Merton, P. A., \& CobB, W. A. (1974). Lambda waves and saccadic suppression [Abstract]. Electroencephalography \& Clinical Neurophysiology, 37, 328.

Musha, T., \& Homma, S. (1990). Do optimal dipoles obtained by the dipole tracing method always suggest true source locations? Brain Topography, 3, 143-150.

NaKamura, A., Kakigi, R, Hoshiyama, M., Koyama, S., Kitamura, Y.,
\& Shimojo, K. (1997). Visual evoked cortical magnetic fields to pattern reversal stimulation. Cognitive Brain Research, 6, 9-22.

PARK, J., \& LeE, C. (2000). Neural discharge coupled to saccade offset in the cat visual cortex. NeuroReport, 11, 1661-1664.

Perez-Borja, C., Chatrian, G. E., Tyce, F. A., \& Rivers, M. H. (1962). Electrographic patterns of the occipital lobe in man: A topographic study based on use of implanted electrodes. Electroencephalography \& Clinical Neurophysiology, 14, 171-182.

Pierrot-Deseilligny, C., Gay mard, B., Müri, R, \& Rivaud, S. (1997). Cerebral ocular motor signs. Journal of Neurology, 244, 65-70.

RÉmond, A., \& Lesèvre, N. (1971). Relationship between responses evoked by moving patterns and lambda responses. Electroencephalography \& Clinical Neurophysiology, 30, 248-254.

Riemslag, F. C.C., van der Heijde, G. L., \& van Dongen, M. M. (1987). Are eye movement evoked potentials different from patternreversal evoked potentials? Documenta Ophthalmologica, 66, 279289.

Roth, M., \& Green, J. (1953). The lambda wave as a normal physiological phenomenon in the human electroencephalogram. Nature, 172, 864-866.

Salzarulo, P., Lairy, G. C., \& Bancaud, J. (1976). Striate cortex potentials related to eye movements in the light and in darkness in the waking human. Perception, 5, 303-308.

Scherg, M., \& Von Cramon, D. (1985). Two bilateral sources of the late AEP as identified by a spatio-temporal dipole model. Electroencephalography \& Clinical Neurophysiology, 62, 32-44.

Scott, D. F., Groethuysen, U. C., \& BickFord, R. G. (1967). Lambda responses in the human electroencephalogram. Neurology, 17, 770-778.

Scott, D. F., Moffett, A., \& Bickford, R. G. (1981). Comparison of two types of visual evoked potentials: Pattern-reversal and eye movement (lambda). Electroencephalography \& Clinical Neurophysiology, 52, 102-104.

Seki, K., Nakasato, N., Fujita, S., Hatanaka, K., Kawamura, T., Kanno, A., \& Yoshimoto, T. (1996). Neuromagnetic evidence that the P100 component of the pattern-reversal visual evoked response originates in the bottom of the calcarine fissure. Electroencephalography \& Clinical Neurophysiology, 100, 436-442.

Shigeto, H., Tobimatsu, S., Yamamoto, T., Kobayashi, T., \& Kato, M. (1998). Visual evoked cortical magnetic response to checkerboard pattern-reversal stimulation: A study on the neural generators of N75, P100 and N145. Journal of Neurological Sciences, 156, 186-194.

Szirtes, J., Marton, M., \& Breuer, P. (1982). Lambda potential in the rhesus monkey. Physiological Psychology, 10, 261-266.

Szirtes, J., \& Marton, M., \& URban, J. (1988). Brain potentials associated with pattern displacement and saccadic eye movements in humans and rhesus monkeys. Acta Physiologica Hungarica, 71, 281302.

Thickbroom, G. W., Knezevic, W., Carroll, W. M.,\& Mastaglia, F. L. (1991). Saccade onset and offset lambda waves: Relation to pattern movement visually evoked potentials. Brain Research, 551, 150-156.

Volkmann, F. C. (1986). Human visual suppression. Vision Research, 26, 1401-1416.

YAGI, A. (1979). Saccade size and lambda complex in man. Physiological Psychology, 7, 370-376.

YAGI, A. (1981a). Averaged cortical potentials (lambda responses) timelocked to onset and offset of saccades. Physiologaical Psychology, 9, 318-320.

YAGI, A. (1981b). Visual signal detection and lambda responses. Electroencephalography \& Clinical Neurophysiology, 52, 604-610.

YAGI, A. (1995). Eye fixation-related potential as an index of visual function. In T. Kikuch, H. Sakuma, I. Saito, \& T. Tsuboi (Eds.), Biobehavioral self-regulation (pp. 177-181). Tokyo: Springer-Verlag.

YAGI, A. (1998). Psychophysical studies of lighting environments. In J. A. Veitch (Ed.), Proceedings of the first CIE symposium on lighting quality (pp. 125-130). Ontario: National Research Council of Canada.

Yagi, A., Imanishi, S., Konishi, H., AKashi, Y., \& Kanaya, S. (1998). Brain potentials associated with eye fixations during visual tasks under different lighting systems. Ergonomics, 41, 670-677.

(Manuscript received September 1, 2000; revision accepted for publication January 9, 2003.) 